\title{
Exploration of the Underlying Nutritional, Inflammatory and Oxidative Stress Pathological Mechanisms in Preeclampsia using Principal Component Analysis
}

\author{
Charles Bitamazire Businge, Benjamin Longo-Mbenza, and Andre Pascal Kengne
}

\section{ABSTRACT}

\begin{abstract}
Normal pregnancy is characterized by a mild systemic inflammatory response and progressive increase in serum inflammatory cytokines that peak in the third trimester. During pregnancy pre-existing inflammatory conditions, acquired oxidative stress arising from the placenta malfunction and nutritional deficiencies can trigger intense systemic responses that lead to endothelial activation, dysfunction and preeclampsia. We investigated the principal nutritional, oxidative and inflammatory pathways that trigger the clinical manifestation of preeclampsia.
\end{abstract}

This case-control study included 250 women with preeclampsia and 150 normotensive pregnant women. Urinary Iodine concentration (IUC) and serum levels of Ferritin, Thyroid-stimulating Hormone (TSH), selenium, Nitric Oxide (NO) gamma glutamyl transferase (GGT), Rheumatoid factor, and high sense C-reactive protein (hs-CRP) of cases and controls were compared using the student's $t$ and the Mann-Whitney $U$ tests. Principal component analysis was carried out to delineate the patterns of association between nutritional, inflammatory and oxidative markers and preeclampsia.

The main pathophysiological pathways identified were the interactions between selenium/iodine deficiency and elevated serum TSH (endothelial dysfunction); serum ferritin, GGT, CRP and low urinary iodine concentration (inflammatory oxidative stress); elevated serum hs-CRP and Rheumatoid factor subclinical inflammation and immune cell activation) and high T3/T4 ratio (acute TSH stimulation of thyroid with low thyroid iodine stores)

Combined selenium and iodine deficiency resulting into elevated TSH, low NO and preferential T3 secretion; acute inflammatory conditions associated with elevated serum GGT, CRP, and Ferritin; and subclinical inflammatory conditions characterized by autoimmunity are some of the major oxidant and inflammatory pathways associated with increased risk of preeclampsia.

Keywords: inflammation, iodine, preeclampsia, oxidative stress, selenium, thyroid-dysfunction.

\section{INTRODUCTION}

The normal physiological changes in pregnancy include mild systemic inflammatory response [1]. This is characterized by an elaboration of inflammatory leukocytes, endothelial activation, the acute phase response, and metabolic features of systemic inflammation, a decrease in plasma albumin levels and increased plasma fibrinogen levels. There is a progressive increase in serum inflammatory cytokines which peak in the third trimester [2], [3]. Redman et al. hypothesized that systemic inflammatory response of preeclampsia is just a more extreme part of the spectrum common to all pregnancies, with preeclampsia developing when the systemic inflammatory process causes maternal Republic of Congo.
Submitted : June 12, 2021

Published : July 05, 2021

ISSN: $2593-8339$

DOI: $10.24018 /$ ejmed.2021.3.4.928

\section{B. Businge}

Department of Obstetrics and Gynaecology, Faculty of Health Sciences, Walter Sisulu University, Private Bag x1 WSU, 5117, Mthatha, South Africa.

Department of Medicine, Faculty of Health Sciences, University of Cape Town, Cape Town, South Africa. (e-mail: bsnbit001@myuct.ac.za / cbusingae@gmail.com / cbusinge@wsu.ac.za) B. Longo-Mbenza

Faculty of Medicine, University of Kinshasa, Democratic Republic of Congo and LOMO University of Research, Kinshasa, Democratic Republic of Congo. longombenza@yahoo.fr)

Andre P. Kengne

Non-Communicable Disease Research Unit, South African Medical Research Council, Cape Town, South Africa. (e-mail: Andre.Kengne ${ }^{\circledR}$ mrc.ac.za)

*Corresponding author (e-mail: longombenza ${ }^{@}$ gmail.com /

systems to decompensate [3]. It is hypothesized that an oxidatively stressed placenta in a previously normal woman, or a normal systemic inflammatory response in a woman with a chronic inflammatory response due to pre-existing chronic infection such as Helicobacter pylori, obesity, essential hypertension, diabetes or other stimulus, can trigger an intense systemic response that leads to endothelial activation, dysfunction and preeclampsia [1].

The aim of the study was to investigate the principal micronutrient, oxidative and inflammatory pathways that are associated with the clinical manifestation of preeclampsia in a peri-urban population of Kinshasa Province, Democratic 


\section{METHODS}

\section{A. Study Design}

This case-control study was carried out as a secondary analysis data of expectant mothers who enrolled as participants of the Communicable Disease, Nutritional, Environmental Epidemiology and Cardio-metabolic Risk Study (CDNECR). Cases were women with preeclampsia, while controls were age-matched pregnant women without preeclampsia. All cases were managed at Maternity Unit of Lomo Medical Centre, Kinshasa, DRC. The controls were women with normal pregnancy that delivered at term at the maternity units of hospitals that referred patients to LMC for the CDNECR study.

\section{B. Ethical Clearance}

The primary study was approved by the Lomo Medical Centre Institutional Review Board (Reference no. LMDE031LMB02). All participants gave informed consent and study procedures complied with the Helsinki Declaration. Further clearance was obtained from the University of Cape Town Human Research Ethics Review Committee (reference number 135/2018).

\section{Study Setting}

The CDNER study was carried out in Kinshasa Province, Democratic Republic of Congo between 2007 and 2008 and coordinated at Lomo Medical Centre. Lomo Medical Centre (LMC) is a tertiary private healthcare centre in the Kinshasa province of the Democratic Republic of Congo (DRC). LMC has a High Dependency Unit with 12 beds that care for patients most of whom are referred from Bondeko clinic, St Joseph Hospital and Ndjili Hospital in Kinshasa Province (Fig. 1). The catchment population is estimated to be about 3 million people. The staff complement is composed of four cardiologists, two Obstetricians and Gynaecologists, three surgeons, two anaesthesiologists, one ophthalmologist, two radiologists, two clinical pathologists and one paediatrician. On average, LMC cares for about 400 complicated maternity cases annually, $80 \%$ of whom are preeclamptic mothers referred for tertiary care.

\section{Sample Size and Derivation of the Analytic Sample}

During the study period, about 2100 antenatal women patients were monitored. One hundred and fifty (150) who delivered at term without developing preeclampsia (normotensive controls) were matched for age with three hundred of the women who developed preeclampsia (cases). However, only 250 cases with complete data were included in the analysis

\section{E. Data Collection}

Preeclampsia was defined according to the International Society for the Study of Hypertension in Pregnancy [4].

Participants were diagnosed with pre-eclampsia when they presented with new onset of hypertension [systolic blood pressure $(\mathrm{SBP})>140 \mathrm{mmHg}$ and or diastolic (DBP) blood pressure $>90 \mathrm{mmHg}$ ] after 20 weeks gestation with proteinuria (spot urine protein/creatinine $>30 \mathrm{mg} / \mathrm{mmol}$, or $>300 \mathrm{mg} /$ day or $2+$ on dipstick testing) or other maternal organ dysfunction: renal insufficiency (creatinine $>90 \mu \mathrm{mol} / \mathrm{L} ; 1.02 \mathrm{mg} / \mathrm{dL}$ ); liver involvement (elevated transaminases at least twice upper limit of normal \pm right upper quadrant or epigastric abdominal pain), neurological complications (altered mental status, blindness, stroke, hyperreflexia, severe headaches, and persistent visual scotomata), haematological complications (thrombocytopenia-platelet count below 150,000/dL, disseminated intravascular coagulation or haemolysis) and uteroplacental dysfunction (foetal growth restriction, abruptio placentae or intrauterine foetal death). Participants were diagnosed with severe pre-eclampsia when they presented with SBP $>160 \mathrm{mmHg}$ or DBP $>110 \mathrm{mmHg}$ with or without systemic organ involvement. Participants were diagnosed with eclampsia when they presented with SBP $>140 \mathrm{mmHg}$ or DBP $>90 \mathrm{mmHg}$ and convulsions after 20 weeks' gestation.

The height and weight were measured according to standardized procedures. Blood pressure was measured according to the American Heart Association guidelines, with the patient's elbow flexed at the heart level. An average of the two measurements with a standard sphygmomanometer taken at intervals of 2 minutes after the participants had been sitting for at least 30 minutes was used [5].

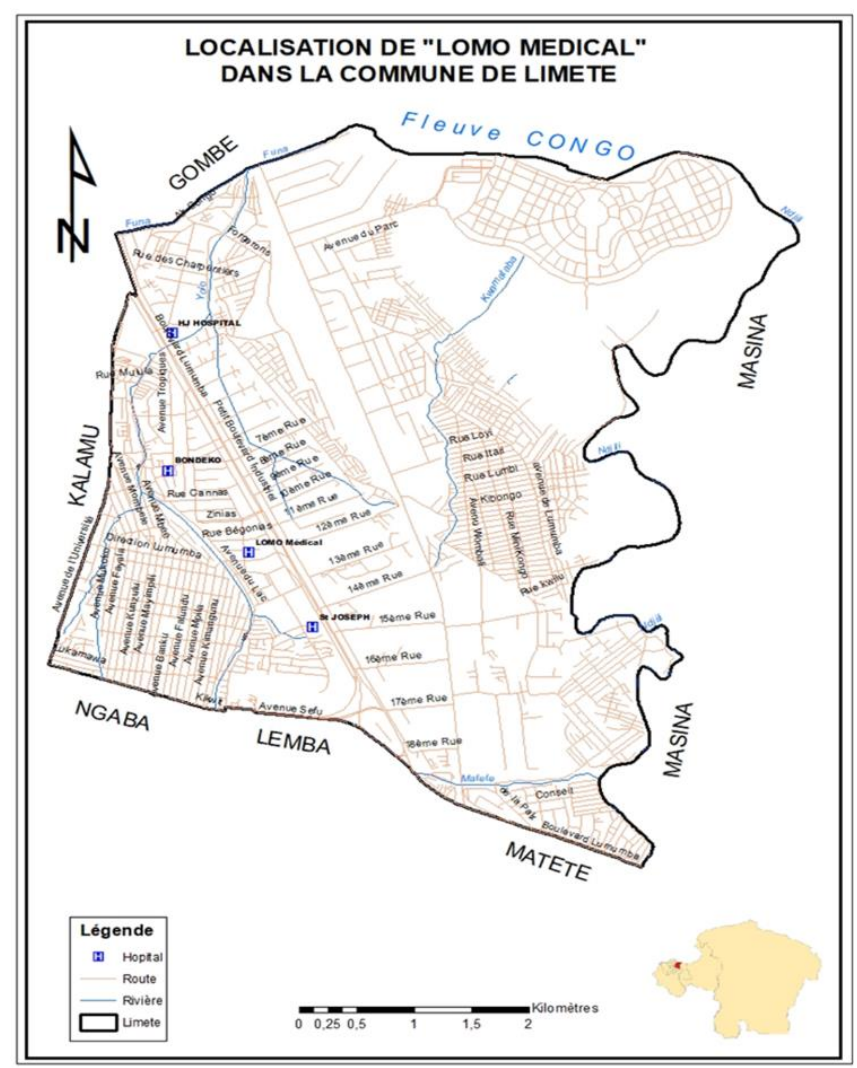

Fig. 1. Map showing the location and catchment area of LOMO Medical Centre and referring Hospitals within Kinshasa Province, Democratic Republic of Congo.

\section{F. Blood Sample Collection and Analysis}

Overnight fasting venous blood was drawn between 7:00 and 9:00 a.m. Fasting serum and plasma samples preserved with ethylenediaminetetraacetic acid (EDTA) and sodium fluoride $(\mathrm{NaF})$ were collected from the cubital fossa. Blood samples were assayed immediately to measure the concentrations serum levels of highly sensitive C-reactive 
protein (hs-CRP), Ferritin, gamma glutamyl transferase (GGT), Rheumatoid factor (RF), C-reactive protein (CRP), thyroid-stimulating hormone (TSH), selenium and nitric oxide (NO). At the same sitting, a mid-stream urine sample was collected to determine urinary Iodine concentration. Serum levels were obtained using calibrated spectrometers and standard routine procedures and specific protocols of manufacturers. TSH were measured by enzyme-linked immunosorbent assay. NO was measured using Cayman kits (Cayman Chemical company Ann Arbot, MI). Urinary iodine concentration (UIC) was measured using the Sandell-Kolthof method.

\section{G. Statistical Analysis}

Categorical variables were compared using chi-square test, while continuous variables were compared using Student's ttest for normally distributed data, and the medians and MannWhitney $U$ test for skewed data. A p-value $<0.05$ was considered as statistically significant. All analyses were performed using the Statistical Package for Social Sciences (SPSS) for windows version 23.0 (SPSS Inc) Chicago, Il, USA. Principal component analysis was carried out to delineate the patterns of association between selected inflammatory and oxidative markers which had significant association with preeclampsia. This statistical tool groups together variables that are strongly correlated with one another and weakly with all the variables in other groups/components. Eigen-values $=1$ were used to identify the key components accounting for the bulk of the observed variance in the manifestation of preeclampsia in the study population. This was to help disaggregate different pathological pathways that may trigger the intense systemic response that leads to endothelial activation, dysfunction and clinical manifestation of preeclampsia.

\section{RESULTS}

\section{A. General Characteristics of the Study Population}

There were 250 cases of preeclampsia with a mean age $32.4 \pm 6$ years and 150 controls cases with a mean age of $33.5 \pm 5.2, p=0.072$. The mean gestational age at recruitment and sample collection was $37.7 \pm 4.0$ weeks of amenorrhoea (WOA) for controls and 31.0 \pm 7.9 WOA for cases $\mathrm{p}<0.0001$. The mean BMI and IgG anti $\mathrm{H}$ pylori for cases and controls respectively were: $25.2 \pm 6.0$ and $22.2 \pm 5.5 \mathrm{~kg} / \mathrm{m}^{2} ; 98.6 \pm 68.0$ and $73.6 \pm 68.1, \mathrm{p}<0.0001$.

\section{B. Median Values and Main Biomarker Components}

Participants with preeclampsia had significantly higher median serum levels of hs-CRP, GGT, Rheumatoid factor, TSH and a significantly higher mean T3/T4 but significantly lower Selenium, NO and urinary Iodine concentration than controls (Table I). Serum Ferritin and CRP were elevated in the lower half of the cases with no significant difference when compared among all cases and controls.

Among the cases, the principal components (suggestive of pathological pathways) (Table II and Fig. 2), with Eigenvalues above 1 , accountings for over 70 percent of the manifestation of preeclampsia were:
Component 1: Low serum Selenium, low NO, low urinary iodine excretion and elevated serum TSH which are features of iodine deficiency, sub-clinical hypothyroidism, and selenium-mediated endothelial dysfunction.

Component 2: Elevated serum Ferritin, GGT, CRP and low urinary iodine concentration which are features of inflammation and oxidative stress.

Component 3: Elevated serum hs-CRP and Rheumatoid factor which are features of inflammation and immune cell activation.

Component 4: high T3/T4 ratio reflecting the increased TSH stimulation of the thyroid in states of chronic iodine insufficiency and low thyroid iodine stores.

TABLE I: MEDIAN/MEAN VALUES OF SELECT OXIDATIVE STRESS AND INFLAMMATORY BIOMARKERS MEASURED IN PARTICIPANTS WITH AND WITHOUT PREECLAMPSIA

\begin{tabular}{|c|c|c|c|}
\hline Variable & $\begin{array}{c}\text { Cases }(\mathrm{n}=250) \\
\text { Median }(\mathrm{p} 25, \mathrm{p} 75) \\
\text { or Mean } \pm \text { SD }\end{array}$ & $\begin{array}{c}\text { Controls }(\mathrm{n}=150) \\
\text { Median }(\mathrm{p} 25, \mathrm{p} 75) \\
\text { or Mean } \pm \text { SD }\end{array}$ & $\mathrm{P}$ value \\
\hline $\begin{array}{l}\text { Selenium } \\
\mu \mathrm{g} / \mathrm{L}\end{array}$ & $9.0(9.0,17.3)$ & $44.0(21.0,102.8)$ & $<0.0001$ \\
\hline $\begin{array}{c}\text { hs - CRP } \\
\text { mg/L }\end{array}$ & $8.0(3.3,9.0)$ & $3.1(2.9,5.0)$ & $<0.0001$ \\
\hline $\mathrm{NO} \mu \mathrm{mol} / \mathrm{L}$ & $2.0(1.0,6.0)$ & $20.8(4.0,43.3)$ & $<0.0001$ \\
\hline $\begin{array}{c}\text { Serum } \\
\text { Ferritin } \mu \mathrm{g} / \mathrm{L}\end{array}$ & $213.0(180.0,345.0)$ & $199.0(167.0,344.0)$ & 0.114 \\
\hline GGT & $99.0(88.0,113.0)$ & $33.0(11.0,99.0)$ & $<0.0001$ \\
\hline $\begin{array}{l}\text { Rheumatoid } \\
\text { Factor }\end{array}$ & $56.0(9.0,88.0)$ & $8.5(7.0,21.5)$ & $<0.0001$ \\
\hline CRP mg/dL & $58.5(39.0,66.0)$ & $57.0(13.0,88.0)$ & 0.437 \\
\hline TSH mIU/L & $6.3(4.1,8.0)$ & $2.5(0.13,4.4)$ & $<0.0001$ \\
\hline $\mathrm{UIC} \mu \mathrm{g} / \mathrm{L}$ & $90.0(78.0,157.2)$ & $351.0(299.0,555.0)$ & $<0.0001$ \\
\hline $\mathrm{T} 3 / \mathrm{T} 4$ ratio & $0.14 \pm 0.05$ & $0.12 \pm 0.00$ & $<0.0001$ \\
\hline
\end{tabular}

$(\mathrm{p} 25, \mathrm{p} 75)=25^{\text {th }}$ and $75^{\text {th }}$ percentiles; $\mathrm{SD}=$ Standard deviation.

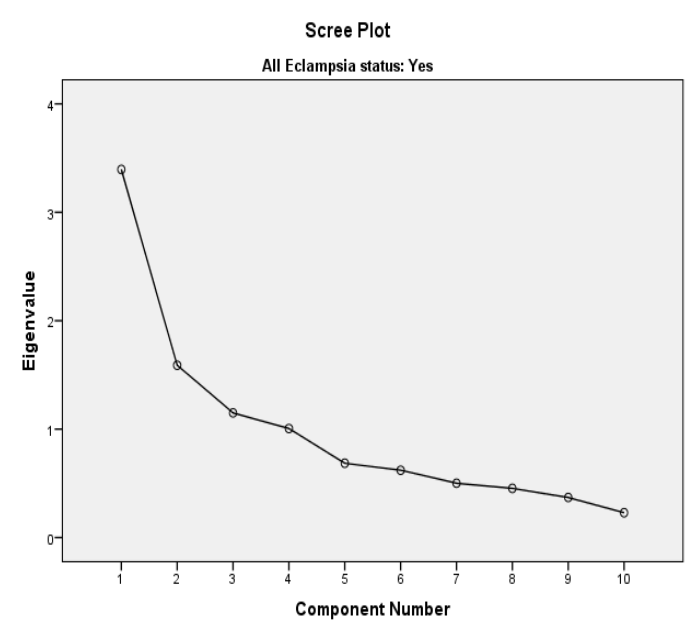

\begin{tabular}{cccc}
\multicolumn{4}{c}{ Components, Eigen-values and variances for cases } \\
\hline \multirow{2}{*}{ Component } & $\begin{array}{c}\text { Eigen } \\
\text { Values }\end{array}$ & $\begin{array}{c}\text { \% of } \\
\text { variance }\end{array}$ & $\begin{array}{c}\text { Cumulative } \\
\%\end{array}$ \\
\hline 1 & $\mathbf{3 . 3 9 5}$ & $\mathbf{3 3 . 9 5 0}$ & $\mathbf{3 3 . 9 5 0}$ \\
2 & $\mathbf{1 . 5 8 9}$ & $\mathbf{1 5 . 8 9 4}$ & $\mathbf{4 9 . 8 4 4}$ \\
3 & $\mathbf{1 . 1 5 0}$ & $\mathbf{1 1 . 4 9 9}$ & $\mathbf{6 1 . 3 4 2}$ \\
4 & $\mathbf{1 . 0 0 6}$ & $\mathbf{1 0 . 0 5 8}$ & $\mathbf{7 1 . 4 0 1}$ \\
5 & .686 & 6.856 & 78.257 \\
6 & .621 & 6.211 & 84.467 \\
7 & .501 & 5.009 & 89.476 \\
8 & .454 & 4.538 & 94.015 \\
9 & .370 & 3.695 & 97.710 \\
10 & .229 & 2.290 & 100.000 \\
\hline
\end{tabular}

Fig. 2. Scree plot showing Eigenvalues for various components of oxidative and inflammatory biomarkers for cases. The Eigenvalues for the 4 major components are shown in bold on the adjacent table. 
TABLE II: ROTATED COMPONENT MATRIX FOR CASES

\begin{tabular}{ccccc}
\hline \multirow{2}{*}{ Biomarker } & \multicolumn{4}{c}{ Component } \\
\cline { 2 - 5 } & 1 & 2 & 3 & 4 \\
\hline Selenium $\mu \mathrm{g} / \mathrm{L}$ & $\mathbf{0 . 8 3 4}$ & -0.184 & -.197 & -.077 \\
NO $\mu \mathrm{mol} / \mathrm{L}$ & $\mathbf{0 . 8 7 0}$ & -0.081 & -.077 & -.055 \\
TSH mIU/L & $\mathbf{- 0 . 7 8 9}$ & 0.097 & -.128 & -.077 \\
UIC $\mu \mathrm{g} / \mathrm{L}$ & $\mathbf{0 . 5 4 1}$ & $\mathbf{- 0 . 4 5 4}$ & -.157 & .061 \\
Serum Ferritin $\mu \mathrm{g} / \mathrm{L}$ & -0.050 & $\mathbf{0 . 7 7 7}$ & -.247 & -.054 \\
GGT & -.239 & $\mathbf{0 . 7 0 3}$ & .336 & .040 \\
CRP mg/dL & -.188 & $\mathbf{0 . 6 9 6}$ & .359 & .119 \\
hs - CRP mg/L & -0.056 & 0.017 & $\mathbf{0 . 8 6 1}$ & -.003 \\
Rheumatoid Factor & -.047 & 0.159 & $\mathbf{0 . 8 1 2}$ & -.032 \\
T3/T4 ratio & -.005 & 0.028 & -.031 & 0.988 \\
\hline
\end{tabular}

The main variables in each component are highlighted.

There were 3 major components (Table III and Fig. 3), with Eigen-values above 1, accounting for over 58 percent variance for a normal pregnancy without manifestation of preeclampsia (the control group) were:

Component 1: high serum Selenium, NO and low TSH which are features of normal thyroid function without thyroid risk of increased thyroid derived superoxide and hydrogen peroxide.

Component 2: normal serum GGT, hs-CRP, CRP and Ferritin depicting absence of systemic inflammation.

Component 3: Normal T3/T4 ratio and normal urinary iodine concentration depicting adequate iodine reserves for normal thyroid function.

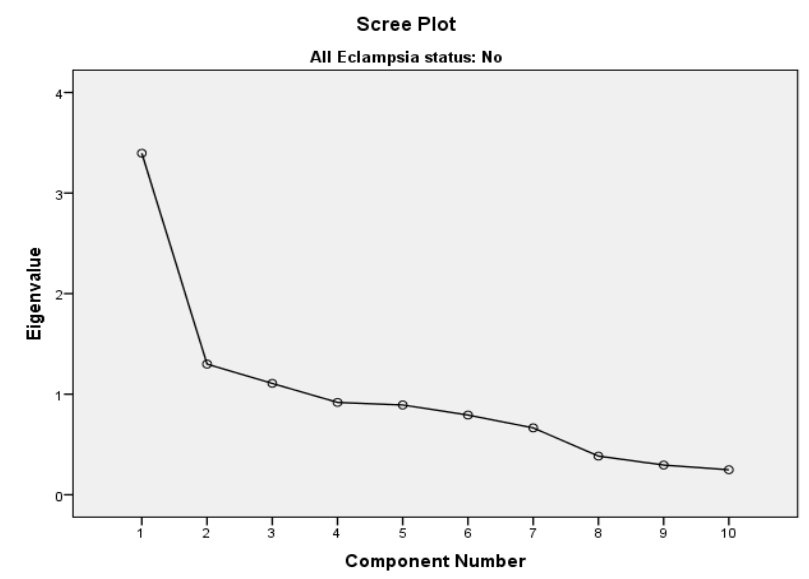

Components, Eigen-values and variances for controls

\begin{tabular}{cccc}
\hline \multirow{2}{*}{ Component } & $\begin{array}{c}\text { Eigen } \\
\text { values }\end{array}$ & $\begin{array}{c}\text { \% of } \\
\text { variance }\end{array}$ & Cumulative\% \\
\hline $\mathbf{1}$ & $\mathbf{3 . 3 9 6}$ & $\mathbf{3 3 . 9 6 2}$ & $\mathbf{3 3 . 9 6 2}$ \\
$\mathbf{2}$ & $\mathbf{1 . 2 9 9}$ & $\mathbf{1 2 . 9 9 3}$ & $\mathbf{4 6 . 9 5 5}$ \\
$\mathbf{3}$ & $\mathbf{1 . 1 0 7}$ & $\mathbf{1 1 . 0 7 3}$ & $\mathbf{5 8 . 0 2 9}$ \\
$\mathbf{4}$ & .918 & 9.184 & 67.213 \\
$\mathbf{5}$ & .893 & 8.928 & 76.141 \\
$\mathbf{6}$ & .793 & 7.926 & 84.068 \\
$\mathbf{7}$ & .665 & 6.650 & 90.717 \\
$\mathbf{8}$ & .384 & 3.842 & 94.560 \\
$\mathbf{9}$ & .296 & 2.956 & 97.515 \\
$\mathbf{1 0}$ & .248 & 2.485 & 100.000 \\
\hline
\end{tabular}

Fig. 3. Scree plot showing Eigenvalues for various components of oxidative and inflammatory biomarkers for controls. Eigenvalues for the 3 major components are shown in bold and are highlighted in the complementary table.
TABLE III: ROTATED COMPONENT MATRIX FOR CONTROLS

\begin{tabular}{cccc}
\hline Biomarker & \multicolumn{3}{c}{ Component } \\
\cline { 2 - 4 } & $\mathbf{0 . 8 3 4}$ & -0.116 & 0.062 \\
Selenium $\mu \mathrm{g} / \mathrm{L}$ & $\mathbf{- 0 . 8 2 4}$ & -0.249 & 0.038 \\
NO $\mu \mathrm{mol} / \mathrm{L}$ & $\mathbf{0 . 8 8 3}$ & 0.096 & 0.048 \\
Baseline TSH mIU/L & 0.128 & $\mathbf{0 . 6 3 1}$ & 0.074 \\
hs - CRP $\mathrm{mg} / \mathrm{L}$ & 0.148 & 0.802 & -0.147 \\
GGT & 0.379 & 0.711 & -0.207 \\
CRP mg/dL & 0.087 & $\mathbf{0 . 5 8 9}$ & 0.151 \\
Serum Ferritin $\mu \mathrm{g} / \mathrm{L}$ & -0.040 & 0.229 & $\mathbf{0 . 7 5 7}$ \\
UIC $\mu \mathrm{g} / \mathrm{L}$ & 0.000 & 0.181 & $\mathbf{- 0 . 6 7 2}$ \\
T3/T4 ratio & 0.411 & 0.318 & -0.006 \\
Rheumatoid Factor & &
\end{tabular}

The main variables in each component are highlighted.

\section{DISCUSSION}

Using the principal component analysis in the current study ten possible mechanisms through which maternal micronutrient, oxidant and inflammatory state increases the risk of preeclampsia were identified. Out of the ten, there were four major contributory pathways in the study population associated with more than $70 \%$ of the cases of preeclampsia. The four were:

Iodine and selenium deficiency acting together with elevated TSH and low serum NO, accounting for almost $34 \%$ of the observed variance among participants with preeclampsia. This matches perfectly with the pathophysiology of thyroid gland in states of combined iodine and selenium deficiency, which is endemic in DRC: iodine deficiency exacerbated by the physiological changes of pregnancy predisposing to low production of thyroxine (T4) and tri-iodothyronine (T3), diminished negative feedback on the pituitary and elevated TSH [6]-[8]. In the thyroid gland, TSH induces Nicotinamide adenine dinucleotide phosphate (NADPH) oxidase, which oxidizes $\mathrm{NADPH}$ to NADP+, liberating superoxide radicals which are then converted to the less potent hydrogen peroxide by Superoxide dismutase, a selenium-dependent enzyme. The hydrogen peroxide not used up in this process, is neutralized by selenium-dependent glutathione peroxidase [9]. Therefore, elevated TSH with simultaneous selenium deficiency will result in excessive production of serum superoxide and hydrogen peroxide by the thyroid gland [9]. The increased circulation of superoxide and hydrogen peroxide radicals which react with Nitric Oxide (NO) forming peroxynitrite which is a more potent oxidant and inducer of inflammation and endothelial dysfunction [9]. This will further diminish NO, accentuating oxidative stress, endothelial dysfunction [9], [10] (hence increasing the risk of preeclampsia. Elevated TSH is also a known stimulant of endothelial dysfunction by inhibition of endothelial NO synthase [11]. This is associated with reduced serum NO and reduction in flow mediated dilatation and endothelial activation, which are well-known features of preeclampsia [12]. TSH by acting on its extra-thyroidal receptors (TSHr) predisposes to endothelial dysfunction and increased carotid media-intima thickness (cIMT) [13], [14]. The increased cIMT is an early marker of atherosclerosis and future cardiovascular disease [14].

The second component was made of elevated serum Ferritin, GGT, CRP and low urinary iodine excretion which are features of inflammation and oxidative stress. Pre- 
pregnancy states such as chronic infections and obesity are associated with elevated serum CRP [15]-[17]. CRP is associated with increased hepcidin production from the liver. Hepcidin is a negative regulator of intracellular iron storage: it leads to reduced iron cellular uptake and increased cellular ferritin secretion by ferroportin, the net effect being an increased serum iron concentration [18]-[20]. GGT is a cell membrane bound enzyme with its active site in the plasma, whose main role is to metabolize reduced extracellular glutathione (GSH) into its precursor amino acids glutamate and cysteine facilitating their re-absorption and intracellular synthesis of GSH [21]. GGT by hydrolysing the glutamyl bond between glutamate and cysteine, produces cysteinylglycine which is subsequently cleaved by dipeptidase into cysteine and glycine. Cysteinyl-glycine and cysteine have been shown to initiate reductive release of iron from its carrier proteins transferrin and ferritin (iron which is physiologically transported in the bound state as $\mathrm{Fe}^{3+}$ is instead released in its bioactive form of $\mathrm{Fe}^{2+}$ ) leading to the Fenton and Harber-Weiss reactions with the release of potent hydroxyl $(\mathrm{OH})$ oxidant radicals [21], [22]. This pathway is further enhanced by low dietary and serum iodine which is exacerbated due physiological changes of pregnancy. Iodine is a potent exogenous scavenger of oxidant radicals, with up to three times the activity of Vitamin C, whose deficiency is likely to significantly alter the oxidation-redox balance, cause endothelial activation and has been found to be associated with increased risk of preeclampsia [23], [24]. Indeed, the placenta is not only a highly metabolic organ with potential for production of oxidant radicals, lipid peroxidation in addition to release of soluble endoglin and soluble fms-like tyrosine kinase 1 in hypoxic states [25]-[28]. It is also one of the organs in the body that concentrates iodine, whose function has hitherto not yet been fully established but could be among others used for scavenging the reactive oxidant radicals [23], [29].

The third principal component encompassed elevated serum hs-CRP and Rheumatoid factor which are features of inflammation and immune cell activation. While CRP is an acute phase protein produced by the liver secondary to any cause of inflammation and has low sensitivity when levels are within normal ranges, hs-CRP has been found to be a sensitive marker of early atherosclerosis and coronary heart disease even when CRP levels are within normal ranges [30]. Thus, the association of hs-CRP and Rheumatoid factor suggests low level chronic inflammation secondary to immune activation. Rheumatoid factors (RF) are a group of antibodies (IgM, IgG or IgA) against the Fc portion of IgG [31]. Although present in $70 \%$ of patients with rheumatoid arthritis, they are not diagnostic for they are also found patients with chronic infections, older people and many autoimmune disorders like systemic lupus erythematosus (SLE) [31]. In the current study, low grade inflammation depicted by elevated hs-CRP and chronic infections depicted by elevated serum Rheumatoid factor may be a result of concurrent central obesity and Helicobacter pylori infection. This may explain the increased risk of preeclampsia among women in the upper normal range of BMI observed in the current study.

The last significant component was characterized by higher T3/T4 ratio among the cases but a lower T3/T4 among controls coupled with normal high urinary iodine excretion reflecting the increased $\mathrm{TSH}$ stimulation of the thyroid in states of chronic iodine insufficiency and low thyroid iodine stores among the cases. Among women with insufficient iodine intake during pregnancy, the transient rise in total T4 secondary to increased thyroid binding globulin (TBG) is relatively low, and this is accompanied by relative reduction in free T4 and T3 of about 30\% after 20 weeks of gestation [32]. If iodine deficiency persists, there is preferential secretion of T3 instead of T4 from the thyroid, resulting in a rise in T3/T4 molar ratio above normal range of $10-22 \times 10^{-3}$. This has been observed in states of persistent thyroid stimulation such as pregnancy and Graves' disease in the presence of depleted intra-thyroidal iodine stores depicting chronic iodine insufficiency [31]. In the current study, this pathway may represent women in the study population with suboptimal iodine intake but adequate selenium intake when compared to those in component one. In these women, the extra-thyroidal stimulation of TSH on TSHr receptors on the endothelium that inhibit NO synthesis and the reduced exogenous anti-oxidant capacity due to iodine deficiency may account for the increased risk of preeclampsia [12], [13]. Our hypothesis is reinforced by the observation in the current study of normal urinary iodine and T3/T4 among controls, which also were grouped together in the resultant principal component analysis.

Although the trigger for clinical manifestation of preeclampsia are placenta derived factors such as soluble Fms-like tyrosine kinase 1 and endoglin which are produced by ischaemic placentae following failure of secondary trophoblastic invasion of myometrial spiral arteries, the severity of preeclampsia seems to depend to some extent on pre-pregnancy maternal oxidative stress and systemic inflammatory state [26], [27]. This inflammatory and oxidative state is upregulated as part of normal physiology of pregnancy which results into early onset preeclampsia in women with high placenta derived factors or late onset preeclampsia, when the normal physiological changes of pregnancy in the third trimester greatly exacerbate the preexisting oxidant and inflammatory state leading to endothelial activation and dysfunction [1]-[3]. The current study used principal component analysis to investigate how different inflammatory and oxidant pathways interact to increase the risk of preeclampsia. The identified prominent role of selenium and iodine deficiency in the process, suggests an opportunity for screening and nutritional intervention in populations with high incidence of preeclampsia. Similarly, our findings of differential role of elevated CRP and hs-CRP, suggest the need to control conditions such as obesity and chronic infections, which are associated with acute and chronic inflammation in women of reproductive age.

\section{STRENGTH AND LIMITATIONS}

This study shows how some micronutrient deficiencies, oxidative stress, acute and chronic inflammatory conditions interact to increase the risk of preeclampsia providing an opportunity for designing prevention measures that are targeted at the identified pathological pathways. 
Although the principal component analysis can be used for delineating how various factors relate to each other during a pathological process, it is not the most appropriate for studying causation. Therefore, some of the observed associations may accrue from preeclampsia other than being factors on the causal pathway to the development of preeclampsia (i.e., reverse causality).

Hence, low dietary intake of iodine and selenium that diminish NO and lead to increased TSH and preferential T3 secretion, acute inflammatory conditions associated with elevated CRP, serum Ferritin and GGT, and subclinical inflammatory conditions characterized by autoimmunity are some of the major oxidant and inflammatory pathways associated with preeclampsia.

\section{ACKNOWLEDGMENT}

The authors thank the Communicable Disease, Nutritional, Environmental Epidemiology and Cardio-metabolic Risk Study team for providing the primary data.

\section{REFERENCES}

[1] C.W. Redman and I.L. Sargent. Preeclampsia and the systemic inflammatory response. Semin Nephrol, vol 24, no. 6, pp. 565-70. 2004.

[2] G.P. Sacks, K. Studena, K. Sargent and C.W. Redman. Normal pregnancy and preeclampsia both produce inflammatory changes in peripheral blood leukocytes akin to those of sepsis. Am J Obstet Gynecol, vol. 179, no. 1, pp. 80-6. 1998.

[3] C.W. Redman, G.P. Sacks and I.L. Sargent. Preeclampsia: an excessive maternal inflammatory response to pregnancy. Am J Obstet Gynecol, vol. 180, no. 2 Pt 1, pp. 499-506. 1999.

[4] A.L. Tranquilli, G. Dekker, L. Magee, J. Roberts, B.M. Sibai, W. Steyn, et al. The classification, diagnosis and management of the hypertensive disorders of pregnancy: A revised statement from the ISSHP. Pregnancy Hypertens, vol. 4, no. 2, pp. 97-104. 2014.

[5] T.G. Pickering, J.E. Hall, L.J. Appel, B.E. Falkner, J.W. Graves, M.N. Hill MN, et al. Recommendations for blood pressure measurement in humans: an AHA scientific statement from the Council on High Blood Pressure Research Professional and Public Education Subcommittee. J Clin Hypertens (Greenwich), vol. 7, no. 2, pp. 102-109. 2005.

[6] D.B. Ngo, L. Dikassa, W. Okitolonda, T.D. Kashala, C. Gervy, J. Dumont, et al. Selenium status in pregnant women of a rural population (Zaire) in relationship to iodine deficiency. Trop Med Int Health, vol. 2, no. 6, pp. 572-81, 1997.

[7] D. Glinoer. The regulation of thyroid function during normal pregnancy: importance of the iodine nutrition status. Best Pract Res Clin Endocrinol Metab, vol. 18, no. 2, pp. 133-52. 2004

[8] M. Zimmermann. The Effects of Iodine Deficiency in Pregnancy and Infancy. Paediatric and perinatal epidemiology, vol. 26, Suppl 1, pp. 108-17. 2012.

[9] P.P. Smyth. Role of iodine in antioxidant defence in thyroid and breast disease. Biofactors, vol. 19, no. 3-4, pp. 121-130. 2003.

[10] 10. Johansen JS, Harris AK, Rychly DJ, Ergul A. Oxidative stress and the use of antioxidants in diabetes: linking basic science to clinical practice. Cardiovasc Diabetol. 2005;4:5.

[11] L. Tian, L. Zhang, J. Liu, T. Guo, C. Gao and J. Ni. Effects of TSH on the function of human umbilical vein endothelial cells. J Mol Endocrinol, vol 52, no. 2, pp. 215-222. 2014.

[12] A. Dardano, L. Ghiadoni, Y. Plantinga, N. Caraccio, A. Bemi, E. Duranti, et al. Recombinant human thyrotropin reduces endotheliumdependent vasodilation in patients monitored for differentiated thyroid carcinoma. J Clin Endocrinol Metab, vol. 91, no. 10, pp. 4175-8. 2006.

[13] E. Lioudaki, N.G. Mavroeidi, D.P. Mikhailidis, E.S. Ganotakis. Subclinical hypothyroidism and vascular risk: an update. Hormones (Athens), vol. 12, no. 4, pp. 495-506. 2013.

[14] D.H. O'Leary and J.F. Polak JF. Intima-media thickness: a tool for atherosclerosis imaging and event prediction. Am J Cardiol, vol. 90 , no. 10c, pp. 181-211. 2002.

[15] E. Lapice, S. Maione, L. Patti, P. Cipriano, A.A. Rivellese, G. Riccardi $\mathrm{G}$, et al. Abdominal adiposity is associated with elevated C-reactive protein independent of BMI in healthy nonobese people. Diabetes Care, vol. 32, no. 9, pp. 1734-6. 2009.

[16] R. Anty, S. Bekri, N. Luciani, M.C. Saint-Paul, M. Dahman, A. Iannelli, et al. The inflammatory C-reactive protein is increased in both liver and adipose tissue in severely obese patients independently from metabolic syndrome, Type 2 diabetes, and NASH. Am J Gastroenterol, vol. 101, no. 8, pp.1824-33. 2006.

[17] WHO. C-reactive protein concentrations as a marker of inflammation or infection for interpreting biomarkers of micronutrient status. Geneva: World Health Organization; 2014.

[18] M.C. Dao, S. Sen, C. Iyer, D. Klebenov and S.N. Meydani. Obesity during pregnancy and fetal iron status: is Hepcidin the link? J Perinatol, vol. 33, no. 3, pp. 177-81. 2013.

[19] V. Manolov, B. Marinov, M. Velizarova, B. Atanasova, V. Vasilev, K. Tzatchev, et al. Anemia in pregnancy and serum hepcidin levels. Int J adv Res, vol 3, no.1, pp.758-61. 2015.

[20] G. Toldi, B. Stenczer, A. Molvarec, Z. Takáts, G. Beko, J. Rigó Jr., et al. Hepcidin concentrations and iron homeostasis in preeclampsia. Clin Chem Lab Med, vol.48, no. 10, pp. 1423-6. 2010.

[21] R. Drozdz, C. Parmentier, H. Hachad, P. Leroy, G. Siest and M. Wellman. gamma-Glutamyltransferase dependent generation of reactive oxygen species from a glutathione/transferrin system. Free Radic Biol Med, vol 25, no. 7, pp. 786-92, 1998.

[22] M. Enoiu, H. Aberkane, J.F. Salazar, P. Leroy, J. Groffen, G. Siest, et al. Evidence for the pro-oxidant effect of gammaglutamyltranspeptidase-related enzyme. Free Radic Biol Med, vol. 29, no. 9 , pp. 825-33, 2000.

[23] R. Winkler, S. Griebenow and W. Wonisch. Effect of iodide on total antioxidant status of human serum. Cell Biochem Funct, vol. 18, no. 2, pp. 143-6. 2000.

[24] M. Gulaboglu, B. Borekci and I. Delibas I. Urine iodine levels in preeclamptic and normal pregnant women. Biol Trace Elem Res, vol. 136, no. 3, pp. 249-57. 2010.

[25] C.Y. Chen, C.P. Chen and K.H. Lin. Biological functions of thyroid hormone in placenta. Int J Mol Sci, vol. 16, no. 2, pp. 4161-79. 2015.

[26] C.W. Redman and I.L. Sargent. Placental debris, oxidative stress and pre-eclampsia. Placenta. 2000;21(7):597-602. 2000.

[27] A. De Vivo, G. Baviera, D. Giordano, G. Todarello, F. Corrado and R. Deanna. PlGF and sFlt-1 as markers for predicting pre-eclampsia. Acta Obstet Gynecol Scand, vol. 87, no. 8, pp. 837-42. 2008.

[28] R. Burns, F. Azizi, M. Hedayati, P. Mirmiran, C. O'Herlihy and P.P. Smyth. Is placental iodine content related to dietary iodine intake? Clin Endocrinol (Oxf), vol. 75, no. 2, pp. 261-4. 2011.

[29] S. Venkatesha, M. Toporsian, C. Lam, J. Hanai J, T. Mammoto, Y.M. Kim, et al. Soluble endoglin contributes to the pathogenesis of preeclampsia. Nat Med, vol.12, no. 6, pp. 642-9. 2006.

[30] T.A. Pearson, G.A. Mensah, Y. Hong and S.C. Smith. CDC/AHA Workshop on Markers of Inflammation and Cardiovascular Disease. Circulation, Vol. 110, no. 25, pp. e543-e4. 2004.

[31] M. Shipley, A. Rahman, D. O'Gradaigh and R. Conway. "Rheumatology and bone disease". In Kumar and Clark's Clinical Medicine. 7th edn. P. Kumar and M. Clark eds. London Elsevier Saunders, 2012, ch 11, pp. 493-560.

[32] D. Glinoer. The regulation of thyroid function in pregnancy: pathways of endocrine adaptation from physiology to pathology. Endocr Rev, vol. 18 , no. 3, pp. 404-33.1997. 